\title{
RELACIÓN ENTRE LA ACTITUD AMBIENTAL Y LA COMPRA DE PRODUCTOS SOCIALMENTE RESPONSABLES EN LOS CONSUMIDORES DE MEDELLÍN, COLOMBIA
}

\author{
RELATION BETWEEN ENVIRONMENTAL ATTITUDE AND THE PURCHASE OF \\ SOCIALLY RESPONSIBLE PRODUCTS IN CONSUMERS IN MEDELLIN, COLOMBIA \\ RELAÇÃO ENTRE ATITUDE AMBIENTAL E A COMPRA DE PRODUTOS \\ SOCIALMENTE RESPONSÁVEIS EM CONSUMIDORES EM MEDELLIN, COLÔMBIA
}

Diana María López Celis; Mónica Eugenia Peñalosa Otero

Magíster en Psicología, Universidad Konrad Lorenz. Docente Asociada II de la Escuela de Administración, Contaduría y Mercadeo, Universidad Jorge Tadeo Lozano. ORCiD: 0000-00027949-0625. E-mail: diana.lopez@utadeo.edu.co, Colombia.

Magíster en Mercadeo Agroindustrial, Universidad Jorge Tadeo Lozano. Docente Asociada II de la Escuela de Administración, Contaduría y Mercadeo, Universidad Jorge Tadeo Lozano. ORCiD: 00000002-2208-9224. E-mail: monica.penalosa@utadeo.edu.co, Colombia.

Recibido: 15 de julio de 2020

Aprobado: 26 de octubre de 2020

DOI: https://doi.org/10.22267/rtend.202102.152

\section{Resumen}

El desarrollo tecnológico mundial ha generado una explosión de consumo, dejando de lado el impacto negativo que este tiene a nivel medio ambiental y sin considerar estrategias para reparar este entorno. Cuando se habla del impacto medioambiental generado por el consumo, es importante entender el consumo sostenible, donde es el consumidor, quien debe responder por el impacto que genera su 
consumo en el medio ambiente; así como el impacto ético generado por las empresas. Este estudio abordó la actitud que los consumidores de la ciudad de Medellín presentan respecto al medio ambiente y, cómo esta se encuentra relacionada con la compra de productos socialmente responsables. Se desarrolló una investigación descriptiva concluyente, con niveles de análisis de corte correlacional, en consumidores mayores de 18 años con muestra controlada por rango de edad, contó con 390 participantes para una confiabilidad del $95 \%$ y $5 \%$ de margen de error. Se determinó una asociación estadísticamente significativa entre las actitudes positivas frente al medio ambiente de los consumidores y la compra de productos socialmente responsables, lo que conlleva al uso de prácticas responsables respecto al medio ambiente, razón por la cual esta ciudad es reconocida por sus avances en gestión ambiental.

Palabras clave: actitud ambiental; comercio; compra responsable; consumo responsable; impacto; medio ambiente; productos; servicios.

JEL: M14; M31; M32; M37; M19; Q56

\begin{abstract}
Global technological development has generated an explosion of consumption, leaving aside the negative impact it has on the environment and without considering strategies to repair this environment. When talking about the environmental impact generated by consumption, it is important to understand sustainable consumption, where it is the consumer who must answer for the impact that their consumption generates on the environment. This study addressed the attitude that consumers in the city of Medellín have towards the environment and how it is related to the purchase of socially responsible products. A conclusive descriptive investigation was carried out, with levels of analysis of correlational cut, in consumers over 18 years with a sample controlled by age range, had 390 participants for a reliability of $95 \%$ and $5 \%$ margin of error. A statistically significant association between the positive attitudes towards the environment of consumers and the purchase of socially responsible products was determined, which leads to the use of responsible practices regarding the environment, which is why this city is recognized for its advances in environmental management.
\end{abstract}

Keywords: environmental attitude; trade; responsible purchasing; responsible consumption; impact; environment; products; services.

JEL: M14; M31; M32; M37; M19; Q56 


\section{Resumo}

O desenvolvimento tecnológico global gerou uma explosão de consumo, deixando de lado o impacto negativo sobre o meio ambiente e sem considerar estratégias para reparar esse ambiente. Ao falar sobre o impacto ambiental gerado pelo consumo, é importante entender o consumo sustentável, onde é o consumidor que deve responder pelo impacto que seu consumo gera no meio ambiente; bem como o impacto ético gerado pelas empresas. Este estudo abordou a atitude que os consumidores da cidade de Medellín têm em relação ao meio ambiente e como está relacionada à compra de produtos socialmente responsáveis. Foi realizada uma investigação descritiva conclusiva, com níveis de análise de corte correlacional, em consumidores acima de 18 anos, com amostra controlada por faixa etária, com 390 participantes, com confiabilidade de $95 \%$ e margem de erro de 5\%. Foi determinada uma associação estatisticamente significativa entre as atitudes positivas em relação ao meio ambiente dos consumidores e a compra de produtos socialmente responsáveis, o que leva ao uso de práticas responsáveis em relação ao meio ambiente, razão pela qual esta cidade é reconhecida por seus avanços em gestão ambiental.

Palavras-chave: atitude ambiental; comércio; compra responsável; consumo responsável; impacto; meio ambiente; produtos; serviços.

JEL: M14; M31; M;32; M37; M19; Q56

\section{Introducción}

El deterioro medio ambiental a nivel mundial desencadena la preocupación y el abordaje del tema en la sociedad, la problemática ambiental no solo se podrá solucionar manipulando únicamente la tecnología, es necesario contribuir al estudio de los valores, actitudes y conductas de las personas con el medio ambiente (Castanedo, 1995). Para Amérigo (2006), en el campo de la psicología social y dada la preocupación por el deterioro ambiental se vienen adelantando diversas investigaciones cuyo fin es encontrar las vías necesarias para lograr transformar las conductas de los individuos como ambientalmente responsables.

En cuanto al concepto de las actitudes en general, Carranza-Vásquez (2016), plantea que estas son las evaluaciones globales y relativamente estables que las personas hacen sobre otras personas, ideas o 
cosas que, técnicamente, reciben la denominación de objetos de actitud (Morales, 2007). Al hablar de actitudes, se hace referencia al grado positivo o negativo con que las personas tienden a juzgar cualquier aspecto de la realidad, convencionalmente denominado objeto de actitud. Las evaluaciones o juicios generales que caracterizan la actitud pueden ser positivos, negativos o neutros, y pueden variar en su intencionalidad o grado de polarización. Del mismo modo, Corral-Verdugo (2010) plantea que las actitudes son propensiones para responder favorable o desfavorablemente a un objeto, evento o situación; estas propensiones están relacionadas de distintas formas con el comportamiento efectivo. Desde la psicología ambiental, Holahan (1996) definió las actitudes como "los sentimientos favorables o desfavorables que inspira un objeto o situación" (p. 115). Debido a que se trata de actitudes hacia el ambiente, normalmente estos sentimientos, creencias o intenciones hacia rasgos u objetos del entorno del sujeto son de naturaleza individual, pero están afectados por las condiciones físicas y sociales que rodean al individuo y su contexto.

En cuanto a la definición de los 2 conceptos psicosociales claves, Amérigo (2006), menciona que la preocupación por el medio ambiente traducida como "actitud ambiental” y la conducta responsable, o "conducta proambiental" serían los términos clave utilizados en la investigación de esta problemática desde la psicología ambiental española.

Con el objetivo de igualar la actitud ambiental con la conducta responsable, tradicionalmente se ha creído que existe una relación entre actitudes hacia la conservación del medio ambiente y un comportamiento similar, sustentable en las personas, sin embargo, existes diferencias para empatar las actitudes, creencias y comportamientos ambientales. Una de las posibles causas queda reflejada en el estudio de Berenguer y Corraliza (2000), en donde consideran que las prácticas de las personas pueden llegar a ser variables en función de una condicionante situacional, por lo que las actitudes en ocasiones no predicen con exactitud las conductas, y en definitiva, concluyen que la preocupación ambiental de forma general puede registrar niveles muy altos, pero no constituye un factor predictor para creencias, valores y comportamientos ecológicos.

Por su parte, para Carranza-Vásquez (2016), las actitudes proambientales constituyen un punto central en el análisis del comportamiento proambiental, debido a que predicen directa o indirectamente la conducta sustentable, de manera que, sin interesar su peso predictivo, ocupan un lugar en los modelos 
que pretenden evaluar e intervenir tanto en el consumo sustentable como en el comportamiento proambiental en general.

Dueñas et al. (2014), advierten sobre la aparición de un nuevo tipo de consumidor, aquel que es consciente de sus actos de compra, tiene unos valores diferentes y se preocupa por sus acciones en el mercado, es bastante extensa en los países desarrollados (Crane y Matten, 2004; Hendarwan, 2002; Rochefort, 1995; Strong, 1996, citados por Dueñas et al., 2014). En este sentido, Newholm y Shaw (2007, p. 255), indican que los consumidores responsables se preocupan por diversos elementos, entre los cuales se encuentran la procedencia del producto, su producción, su manufactura, los regímenes opresivos, los derechos humanos, las relaciones laborales, el desarrollo armamentista de los países, el uso experimental de animales y las donaciones políticas. En este sentido, la preocupación por productos o servicios que provengan de producciones no sostenibles, así como los efectos de los productos o servicios sobre el medio ambiente y sobre las personas, se vuelve relevante para este tipo de consumidores, incluso si estos factores trascienden el contexto nacional y ocurren en un contexto internacional (Llopis, 2009).

\section{Referentes teóricos}

\section{Consumo Responsable}

En la década de 1970, el concepto de consumo verde fue referencia para interrelacionar diferentes disciplinas, entre ellas, la ecológica y el mercadeo, lo cual permitió el desarrollo de la investigación en problemáticas ambientales, de esta manera surgen nuevos conceptos como consumo sostenible o responsable (Dueñas et al., 2014, citados por Pascual et al., 2015). El consumo verde según Elkington y Hailes (1989, p. 235), es aquel que evita productos que ponen en riesgo la salud del consumidor o de otro; causan daño significativo al medioambiente durante la manufactura, su uso o desperdicio; consumen una cantidad desproporcionada de energía; causan un desperdicio innecesario; usan materiales derivados de especies o ambientes amenazados; así como aquellos que implican un maltrato innecesario de animales o que de manera adversa afectan a otros países. Esta noción sobre el consumo verde se amplió rápidamente debido a que el aspecto ético y moral aparecía evidente en la toma de decisiones de los compradores, ampliándose el concepto al de consumidores éticos. 
Esta situación permite incorporar elementos éticos y morales en la conducta del consumidor, en la que satisface una necesidad, pero va más allá de una condición de mercado para analizar condiciones fuera de este, desde el punto de vista del activismo medioambiental, como consciente de una realidad en crisis ambiental. En este sentido, tanto para Shaw y Shiu (2002) y Dueñas et al. (2014), la decisión del consumidor ético se convierte en un elemento de transformación e impacto en la sociedad que depende de la conservación de los recursos naturales, pasando de un concepto antiguo de solo producción a un escenario de comportamientos colectivos para configurar un concepto más integral en un consumo socialmente responsable (CSR) (Pascual et al., 2015).

De acuerdo con Barragán y Ayaviri (2018), la mayoría de las grandes empresas en la actualidad tienen políticas ambientales, adoptan la teoría del desarrollo sostenible y aspiran a ser corporaciones socialmente responsables. Sin embargo, los críticos sostienen que; incluso el marketing social es una herramienta utilizada por las compañías para no mejorar el bienestar social, sino para lograr una ventaja competitiva con el objetivo de crear riqueza para los propietarios (Roper y Parker, 2013). Desgraciadamente hoy en día las consideraciones que apelan al consumo responsable, o bien al comercio justo, siguen considerando de alguna manera que la responsabilidad es individual y no eminentemente social (Gil, 2008).

La formación de una actitud ambiental sustentada en las prácticas cotidianas y en la importancia que el individuo construye frente al tema ambiental, así como en las relaciones con los productos que consume, entre otros; tiene que ver no solo con el consumo responsable; sino con el consumo sostenible, el cual, parte de entender las prácticas de consumo desde una variable de consciencia frente a lo que se compra y lo que se consume, desde el análisis casi cotidiano y aprendido de los impactos que se tiene sobre el medio ambiente.

\section{Educación Sostenible}

Los problemas ambientales y la escasez de recursos naturales en diferentes partes del mundo han generado una preocupación social y ha despertado el interés por el consumo de las personas y la actitud ambiental de estas; entendida como una predisposición aprendida, que impulsa al individuo a comportarse de una manera consistentemente favorable o desfavorables en relación con un objeto determinado (Schiffman y Kanuk, 2010). Por este motivo, uno de los principales objetivos a nivel 
mundial es la educación sostenible, con la cual se busca educar a los ciudadanos a construir un mundo sostenible y saludable que brinde una calidad de vida mejor y justa para todos, siendo la educación la herramienta adecuada para generar en las personas un cambio, desarrollando conciencia amigable y responsable con el medio ambiente. Este aspecto ha permitido también, el fortalecimiento del concepto de consumo responsable, pretendiendo generar conciencia ambiental en las personas para que sean más analíticas y críticas frente al consumo y desecho de los productos de compra.

\section{Compra Socialmente Responsable}

Para Morillo (2008), la visión de RSC orienta la gestión del marketing tomando en cuenta, en primer lugar, el objetivo de satisfacer necesidades de manera segura y responsable al considerar los beneficios o impactos negativos generados a su entorno (clientes internos, mercado y sociedad). Es así como uno de los conceptos más recientes de la mercadotecnia, según lo señalan Kotler (2005), establece que la organización debe determinar las necesidades, los deseos e intereses de los mercados meta y entregar los satisfactores deseados de manera más efectiva y eficiente que la de los competidores, pero de tal manera que mantenga o mejore el bienestar del consumidor y de la sociedad.

En la actualidad, se pueden evidenciar dos actitudes de compra distintas. De un lado, el consumidor que compra de acuerdo con lo que considera socialmente aceptable, sin importar el aspecto ético, imperando en mayor medida la relación calidad-precio; y de otro lado, aquel consumidor que dentro de esas intenciones de compra también afecta aspectos externos a la relación calidad-precio manejando conceptos asociados al consumo responsable y presentando mayor empatía a la hora de seleccionar los productos. Lo anterior, es considerado como una nueva forma de comprar y consumir, debido al cambio en los hábitos de compra y de consumo, hacia los productos responsables o eco amigables.

Existen distintos modos de estudiar este tipo de diferencia en los consumidores. La elección del consumidor no solo consta de las variables propias de la psicología del consumidor como la calidad y el precio, sino también del impacto ambiental y social que tiene el producto a comprar. (Fundación para la Educación Superior y el Desarrollo [Fedesarrollo], 2019). Otras investigaciones, reflejan las razones por las que otros factores entran a ser parte de esas intenciones, tales como factores externos 
a la relación calidad-precio, encontrando dos líneas de investigación. La primera busca predecir el comportamiento del consumo responsable, identificando las intenciones de uso y compra, mientras la segunda está enfocada en identificar las causas que actúan directa o indirectamente en la actitud de las personas que manifiestan una intención de compra.

\section{Actitud Ambiental}

Las actitudes o intenciones frente al problema ambiental derivado de consumo no siempre son consistentes con los hábitos o los comportamientos ambientalmente responsables; por tanto, es importante definir el término actitud ambiental para poder determinar qué tipos de consumidores existen de acuerdo a esta. Desde la Psicología Ambiental, Holahan (1991, p. 15) la define como "los sentimientos favorables o desfavorables que se tienen hacia alguna característica del medio ambiente o hacia un problema relacionado con él"; sin embargo, las personas sólo realizan conductas ambientalmente responsables cuando se encuentran informados sobre la problemática ambiental, lo cual hace que se motiven y se vean capaces de generar cambios cualitativos; en otras palabras, el consumidor responsable es una persona informada y consciente de sus hábitos de consumo. Además de conocer y exigir sus derechos como consumidor, busca la opción de consumo con el menor impacto negativo posible sobre el medio ambiente y con un efecto positivo en la sociedad. Esta manera responsable de consumir se traduce en muchos pequeños actos y decisiones diarias, y puede llegar a atravesar, todos los ámbitos de la vida (Natalichio, 2011).

Corral-Verdugo y de Queiroz (2004) señalan que, la conducta proambiental es el conjunto de acciones deliberadas y efectivas que responden a requerimientos sociales e individuales que se reflejan en la protección del medio. Por ello, es preciso fomentar la realización de comportamientos más respetuosos con el medio ambiente, según Durán et al. (2007), resaltan la obligación de conocer todos los elementos que puedan contribuir a esa finalidad.

Actualmente tanto el consumidor como el sector empresarial se han ido concientizando acerca del consumo debido, entendido como aquel que tiene un lugar preponderante en la vida cotidiana y también, en la actual crisis ambiental. Para Fedesarrollo (2019), además del incremento de actividades productivas, el consumo aporta a uno de los mayores problemas ambientales, entre los que se encuentran el manejo y disposición final de los residuos sólidos; por tanto, en la medida en que las 
empresas y los consumidores sean conscientes del rol que ocupan, se encaminarán esfuerzos para minimizar el impacto negativo que las decisiones individuales tienen sobre el bienestar general.

\section{Actitud y conducta de compra ambiental en consumidores de Medellín}

Medellín es una ciudad colombiana que económicamente sobresale como uno de los principales centros financieros, industriales, comerciales y de servicios del país, en especial en los sectores de economía textil, confecciones, metalmecánico, eléctrico y electrónico, telecomunicaciones, automotriz, alimentos y salud, entre otros, considerados como focos de industrialización. En la actualidad, y de acuerdo con el Plan Ambiental Municipal PAM: 2012 - 2019 "hacia una ciudad sostenible”, la Alcaldía de Medellín (2011) viene implementando un plan ambiental encaminado al fortalecimiento de una ciudad sostenible; donde se involucra la empresa privada, lo cual hace que Medellín sea foco de investigaciones para revisar el impacto de la implementación de estos planes como referente en el tema.

Podemos afirmar que de acuerdo con diversos estudios se demuestran actitudes positivas del consumidor respecto al medio ambiente y al consumo de productos proambientales. Según Fedesarrollo (2019), estos consumidores, se puede evidenciar que la relación ambiental estaba en el centro de la intencionalidad de ciertas decisiones de compra y desechos que estarían en el origen de lo que podría considerarse como ciudadanos proambientales.

En el mismo sentido, Peñalosa y López (2018), argumentan que, en cuatro capitales colombianas estudiadas entre las que se encuentra la ciudad de Medellín, existe una actitud positiva con respecto al consumo responsable, lo que permite al consumidor realizar compras con más sentido de responsabilidad en cuanto al impacto que estas conductas pueden tener, no solo para ellos, sino para su entorno.

A la luz de lo expuesto en la revisión de la literatura, se plantea la siguiente pregunta de investigación: ¿Cuál es la relación entre la actitud ambiental y la compra de productos socialmente responsables, en los consumidores de Medellín Colombia? 


\section{Hipótesis de trabajo}

La actitud ambiental y la compra de productos socialmente responsables guardan asociaciones de dependencia estadísticamente significativas.

\section{Metodología}

\section{Variables de la investigación}

\section{Variable Predictora}

Actitud positiva o negativa frente al medio ambiente, evaluada a través de frases actitudinales en una escala de Likert de 4 puntos donde 1; es totalmente de acuerdo, 2 es de acuerdo, 3 en desacuerdo y 4 totalmente en desacuerdo, estas medidas fueron anidadas a través del método top two box y bottom two, las dos medidas más altas ( 1 y 2) se unen y van a la caja de top two box; y por otro lado, se unen las medias más bajas (3 y 4) y quedan en la caja bottom two box, sin dejar de considerar el sentido de cada frase actitudinal, lo cual hace que la actitud positiva o negativa dependa precisamente del sentido de la frase y no necesariamente coincida la actitud positiva con la medida anidada en la caja de top two box, es posible que se encuentre en la caja bottom two box o viceversa, obteniendo de esta manera una variable en escala ordinal, se utiliza éste método para evitar el sesgo al tomar solamente las medidas extremas, eliminando la acidez de la prueba y suavizando la calificación.

\section{Variable Criterio}

Compra de productos socialmente responsables, medida en una escala nominal donde 1 es interpretado como la compra que alguna vez han realizado los consumidores de Medellín de productos socialmente responsables y 2 , los consumidores nunca han realizado compras socialmente responsables.

\section{Tipo de Investigación}

Se realizó una investigación de alcance descriptivo concluyente, con niveles de análisis de corte correlacional y enfoque cuantitativo. 


\section{Diseño Muestral}

Muestreo no probabilístico, con una muestra de 390 consumidores de la ciudad de Medellín, mayores de 18 años que hubieran realizado algún tipo de compra para el consumo del hogar o consumo personal en hipermercado, supermercado, autoservicio, minimercado de barrio o tienda de barrio durante el último mes. Se controló la variable edad por rangos, de acuerdo con la distribución poblacional; 18 a 25 años 34\%, 26 a 35 años 22\%, 36 a 45 años 24\%, 46 a 60 años $16 \%$ y más de 60 años $4 \%$. Con una confiabilidad del $95 \%$ y $5 \%$ de margen de error.

Cabe anotar que, se presenta la participación del estudio por rangos de edades como soporte de que la muestra tomó diferentes rangos poblacionales y se trató de tener en cuenta de acuerdo a la distribución poblacional, para que hubiera representatividad poblacional, sin embargo, el objeto del estudio no es el de realizar análisis por rangos e edades, teniendo en cuenta que en caso de hacerlo la muestra del estudio se bajaría mucho.

\section{Técnica de Recolección de Datos}

La revisión de la literatura llevó a definir y obtener las variables utilizadas, las cuales fueron consideradas como una adaptación al instrumento propuesto por Castanedo (1995), sobre la valoración de las cualidades a favor del medio ambiente (EAPA), la cual ha sido validada mediante el análisis factorial y es confiable de acuerdo con el indicador de Alpha de Cronbach. Las variables están redactadas para acordar la etapa actual sobre las actitudes ambientales en el ámbito del social y calcular como son observadas las personas a través del contacto ambiental (Castanedo, 1995, p. 258).

En total la escala contiene 17 ítems, las escalas utilizadas fueron conservadas y son del tipo Likert, de modo que para cada encuestado existan distintas alternativas graduadas en intensidad de 4 niveles, en una escala de likert (Alaminos y Castejón, 2006) donde; muy de acuerdo=1, de acuerdo=2, en

desacuerdo=3 y muy en desacuerdo=4. Con esta escala se construyó un cuestionario estructurado, implementado durante el segundo semestre de 2019, auto administrado vía online. 


\section{Tabla 1}

\section{Discusión}

\section{Resultados del análisis de contingencia Chi cuadrado}

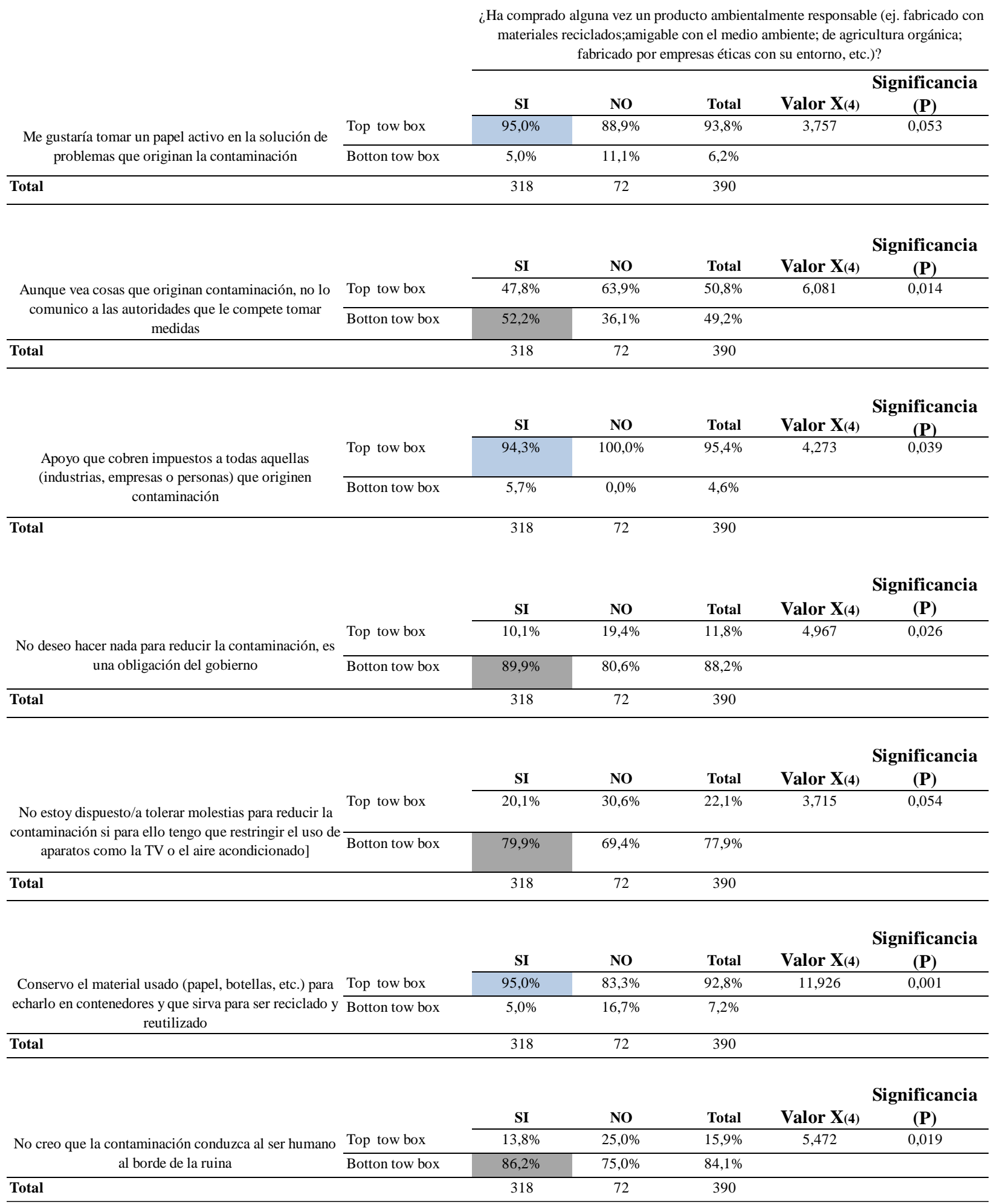

Fuente: elaboración propia. 
Se utilizó la prueba de chi $^{2}$; la cual es una prueba, que sirve para someter a prueba de hipótesis distribuciones de tablas de frecuencias con variables en escala nominal y ordinal, a través de la cual se encontró asociación estadísticamente significativa a un nivel de p: 0,005 entre la variable predictora; Actitud frente al medio ambiente y las variables criterio Compra de productos socialmente responsables.

De acuerdo al análisis realizado, se encontró asociación estadísticamente significativa entre los compradores de algún producto ambientalmente responsable en Medellín, con niveles de significancia por debajo de 0,05, y una actitud positiva con porcentajes actitudinales por encima del 90\%, en el nivel de top two box (totalmente de acuerdo y de acuerdo), mostrando una actitud positiva frente a tomar un papel activo en la solución de problemas que originan la contaminación 95\%, apoyo del cobro a personas o empresas que originen contaminación 94,3\%, y el reciclaje al depositar material usado en contenedores para ser reutilizado. Y niveles por encima del 50\% en el nivel Botton two box (totalmente en desacuerdo y desacuerdo); consideran que deben denunciar ante las autoridades competentes las cosas que originan contaminación $52,2 \%$, piensa que es su responsabilidad, no solamente le corresponde al gobierno hacer algo para reducir la contaminación 89,9\%, están dispuestos a tolerar molestias si con esto consiguen reducir la contaminación 79,9\% y son consciente que la contaminación puede llevar a la humanidad a la ruina $86,2 \%$.

En términos generales, cabe anotar que las actitudes positivas frente al medio ambiente en los consumidores de Medellín, acompañan la compra de algún producto socialmente responsable como lo muestra la tabla de resultados, lo cual contradice algunas apreciaciones de la literatura existente sobre la asociación entre actitudes positivas frente al medio ambiente y la compra de productos socialmente responsable, como en el caso de Berenguer y Corraliza (2000), quienes en su estudio concluyen que la preocupación ambiental de forma general puede registrar niveles muy altos, pero no constituye un factor predictor para creencias, valores y comportamientos ecológicos, puesto que el presente estudio encontró que para los consumidores de Medellín existe asociación entre estas dos variables. 
Por otro lado, están los estudios que confirman los resultados de esta investigación como CarranzaVásquez (2016), quien concluye que las actitudes ambientales deberán ser un eje central en el estudio del comportamiento proambiental, debido al factor predictivo en la conducta sustentable.

Así mismo, (Dueñas et al., 2014), indica que ha aparecido un nuevo tipo de consumidor, con características de conciencia en sus actos de compra, quienes tiene valores diferentes y, por ende, inquietudes en sus acciones en el mercado del consumo.

Los estudios desarrollados sobre esta misma ciudad por Fedesarrollo (2019) y Peñalosa y López (2018); recalcan el cómo sin desconocer los resultados en cada población estudiada, definitivamente dependerán de las condiciones situacionales respecto al tema medioambiental, las cuales van de la mano con las políticas impartidas tanto por el gobierno como por la industria privada, sumada a sus estrategias de difusión puesto que las personas sólo realizan conductas ambientalmente responsables cuando se encuentran informados sobre la problemática ambiental, aspecto que favorece a la ciudad de Medellín para haber encontrado asociación positiva y significativa entre las variables de estudio. Al evidenciar en la ciudad de Medellín el uso de estas prácticas responsables respecto al medio ambiente, se ratifica como esta ciudad es reconocida por sus avances en gestión ambiental.

\section{Conclusiones}

De acuerdo con la investigación podemos concluir que en el repertorio conductual de los consumidores en los últimos años, hoy se evidencian nuevas variables a considerar desde la perspectiva del consumidor al seleccionar un producto, dado por el cambio de actitud en algunas de las nuevas generaciones poblacionales (por ejemplo los millennials o centennials), que aunque no se contemplaron en el marco teórico, son generaciones en donde la variable medio ambiental cobra gran relevancia en sus estilos de vida y hábitos de compra; por otra parte, el estudio deja abiertas las puertas para hacer la revisión en otras regiones, puesto que, el estudio evalúa una región específica de Colombia y al analizar otras regiones del mismo país o en otros países podría cambiar los resultados debido a patrones en estilo de vida, culturales, políticas de gobierno, y los hábitos de consumo de los consumidores; así mismo las estrategias respecto al consumo responsable, y específicamente frente al factor medio ambiental, desarrolladas por las marcas en cada zona geográfica. 
Relación entre la actitud ambiental y la compra de productos socialmente responsables en los consumidores de Medellín, Colombia

Diana María López Celis; Mónica Eugenia Peñalosa Otero

Estos hallazgos abren las puertas para contemplar la implementación de estrategias de mercadeo basadas en variables de consumo responsable, específicamente las variables ambientales, así como la oportunidad de explorar si las variables sociales y éticas impactan al consumidor de la misma manera, y pueden también ser usadas en las estrategias a desarrollar para lograr un vínculo entre el consumidor, la marca y la responsabilidad compartida por el cuidado del medio ambiente.

\section{Referencias}

(1) Alaminos, A. y Castejón, J. L. (2006). Elaboración, análisis e interpretación de encuestas, cuestionarios y escalas de opinión. Marfil.

(2) Alcaldia de Medellín - Secretaría del Medio Ambiente. (2011). Plan Ambiental Municipal PAM: $2012 \quad-\quad 2019 \quad$ Hacia Una Ciudad Sostenible. https://www.medellin.gov.co/irj/go/km/docs/wpccontent/Sites/Subportal\%20del\%20Ciudadano/ Medio\%20Ambiente/Secciones/Publicaciones/Documentos/2012/PAM/TOMO\%20IV.\%20INF ORME\%20EJECUTIVO.pdf

(3) Amérigo, M. (2006). La investigación en España sobre actitudes proambientales y comportamiento ecológico. Medio ambiente y comportamiento humano, 7(2), 45-71. http://mach.webs.ull.es/PDFS/Vol7_2/Vol7_2_c.pdf

(4) Barragán, M. y Ayaviri, D. (2018). Ética del Consumo en la Gestión de la Seguridad Alimentaria en el Cantón Santo Domingo de los Colorados, Ecuador. Información Tecnológica, 29(5), 143156. http://dx.doi.org/10.4067/S0718-07642018000500143

(5) Berenguer, J. y Corraliza, J. (2000). Preocupación ambiental y comportamientos ecológicos. Psicothema, 12(3), 325-329.

(6) Carranza-Vásquez, L. (2016). Diferencias entre las actitudes proambientales y el consumo de servicios públicos (agua y energía eléctrica) en estratos 2 y 5 de la ciudad de Bogotá. Revista Perspectiva Empresarial, 3(2), 83-94. https://doi.org/10.16967/rpe.v3n2a7

(7) Castanedo, C. (1995). Escala para la evaluación de las acittudes pro-ambientales (EAPA) de alumnos universitarios. Revista Complutense de Educación, 6(2), 253-278.

(8) Corral-Verdugo, V. y de Queiroz, J. (2004). Aproximaciones al estudio de la conducta sustentable. Medio Ambiente y Comportamiento Humano, 5(1y2), 1-26. 
(9) Corral-Verdugo, V. (2010). Psicología de la sustentabilidad. Un análisis de los que nos hace pro ecológicos y pro sociales. Trillas.

(10) Dueñas, S., Perdomo-Ortiz, J. y Villa, L. (2014). El concepto de consumo socialmente responsable y su medición. Una revisión de la literatura. Estudios Gerenciales, 30(132), 287-300. https://doi.org/10.1016/j.estger.2014.01.022

(11)Durán, M., Alzate, M., López, W. y Sabucedo, J. (2007). Emociones y comportamiento proambiental. Revista Latinoamericana de Psicología, 39(2), 287-296.

http://pepsic.bvsalud.org/scielo.php?script=sci_arttext\&pid=S012005342007000200006\&lng=pt\&tlng=es

(12) Elkington, J., \& Hailes, J. (1989). The green consumer guide: From shampoo to champagne: highstreet shopping for a better environment. V. Gollancz.

(13)Fundación para la Educación Superior y el Desarrollo [Fedesarrollo]. (2019). Encuesta de Opinión del Consumidor. https://www.fedesarrollo.org.co/tipo-de-encuesta/consumidor-eoc

(14) Gil, A. (2008). Por una ética del consumo política: Florence Kelley y la Liga de Consumidores. Athenea Digital, $\quad$ 311-316. http://psicologiasocial.uab.es/athenea/index.php/atheneaDigital/article/view/584

(15) Holahan, C. (1991) Psicología Ambiental. Un Enfoque General. Limusa.

(16) Holahan, C. (1996). Psicología ambiental. Un enfoque general. Limusa.

(17) Kotler, P. (2005). Las preguntas más frecuentes sobre Marketing. Grupo Editorial Norma.

(18)Llopis, R. (2009). Consumo responsable y globalización reflexiva: un estudio referido al comercio justo en España. Revista Española del Tercer Sector, (11), 145-165.

(19) Morales, F. (2007). Psicología social (3 ${ }^{a}$.ed.). McGraw-Hill.

(20) Morillo, M. (2008). Hacia una gestión de marketing socialmente responsable: Decisiones de compra y de comunicación. Economía, 189-206. https://www.redalyc.org/pdf/1956/195617231009.pdf

(21) Natalichio, R. (2011). Con-sumo cuidado, Comercio justo y consumo responsable. Revista Fusion. https://www.revistafusion.com/201106242141/Medio-Ambiente/Medio-Ambiente/consumo-cuidado-comercio-justo-y-consumo-responsable.htm

(22) Newholm, T., \& Shaw, D. (2007). Studying the ethical consumer: A review of research. Journal of Consumer Behaviour, 6(5), 253-270. https://doi.org/10.1002/cb.225 
Relación entre la actitud ambiental y la compra de productos socialmente responsables en los consumidores de Medellín, Colombia

Diana María López Celis; Mónica Eugenia Peñalosa Otero

(23) Pascual, M., Peñalosa, M. y López, D. (2015). El consumo socialmente responsable en el mercado colombiano. Cuadernos Latinoamericanos de Administración, 11(20), 61-70. https://doi.org/10.18270/cuaderlam.v11i20.624

(24) Peñalosa, M. y López, D. (2018). El estereotipo de los colombianos frente al consumo socialmente responsable. Pensamiento \& Gestión, (44), 243-260. https://dx.doi.org/10.14482/pege.44.10384

(25) Roper, S., \& Parker, C. (2013). Doing well by doing good: A quantitative investigation of the litter effect. Journal of Business Research, 66(11), 2262-2268. https://doi.org/10.1016/j.jbusres.2012.02.018

(26) Schiffman, L. y Kanuk, L. (2010). Comportamiento del consumidor. (10. ${ }^{\mathrm{e} e d .}$.). Pearson.

(27) Shaw, D., \& Shiu, E. (2002). An assessment of ethical obligation and self-identity inethical consumer decision-making: A structural equation modelling approach. International Journal of Consumer Studies, 26(4), 286-293. https://doi.org/10.1046/j.1470-6431.2002.00255.x

Cómo citar este artículo: López, D. y Peñalosa, M. (2021). Relación entre la actitud ambiental y la compra de productos socialmente responsables en los consumidores de Medellín, Colombia. Tendencias, 22(1), 1-17. https://doi.org/10.22267/rtend.202102.152 